Invest. Pens. Crit.(ISSN 1812-3864; eISSN 2644-4119)

Vol. 9, No. 2, Mayo - Agosto 2021.pp. $04-15$

DOI: https://doi.org/10.37387/ipc.v9i2.231

Artículo Científico

\title{
Dislexia, rendimiento lector y su relación con la inequidad y el desempeño escolar
}

\author{
Dyslexia, reading performance and its relationship with inequity and school \\ performance.
}

\author{
Daniel Cubilla-Bonnetier, ${ }^{1,}$, , Abdel Solís-Rodríguez ${ }^{2}$, Francisco Farnum ${ }^{4}$, \& Jessibeth Caballero ${ }^{3}$ \\ ${ }^{1}$ Universidad Católica Santa María la Antigua (USMA), Panamá. \\ ${ }^{2}$ Universidad Católica Santa María la Antigua (USMA), Panamá. Sistema Nacional de Investigación (SNI), SENACYT, Panamá. \\ ${ }^{3}$ Universidad de Panamá \\ ${ }^{4}$ Instituto de Salud Global y Medicina Tropical, Universidad Católica Santa María la Antigua (USMA), Panamá.
}

*Autor para correspondencia: dcubilla@usma.ac.pa

Recibido: 17 de noviembre de 2020

Aceptado: 24 de marzo de 2021

\section{Resumen}

Se ha descrito la relación existente entre rendimiento lector y desempeño académico, así como el impacto que sobre ambos tiene la vulnerabilidad socioeconómica del niñola. No existe, en cambio, abundancia de literatura sobre el impacto del nivel socioeconómico en la presencia de dislexia del desarrollo. Sobre una muestra de 216 escolares de Educación Primaria de escuelas públicas y particulares de la Ciudad de Panamá con la presencia de 12 participantes con dislexia, se estudiaron las variables de rendimiento lector, categoría socioeconómica y rendimiento académico. Aunque no se encontró en el presente estudio una relación directa entre el nivel socioeconómico de los participantes y la prevalencia de dislexia, pareciera que el grupo situado en la parte más baja de la escala ve triplicadas sus probabilidades de presentar el trastorno, en comparación con el resto de la muestra. Los resultados indican una relación más lineal entre el nivel socioeconómico y el rendimiento lector, así como el impacto negativo que la dislexia y la baja competencia lectora tienen sobre el rendimiento académico. Se propone la hipótesis de que los resultados podrían concordar con la presencia en el trastorno disléxico de un componente neurobiológico y genético, pero cuyo impacto podría ser modulado en intensidad por el componente ambiental y se reflexiona sobre la porción de bajo rendimiento escolar que podría evitarse con políticas activas de abordaje de la dislexia.

Palabras Clave: Dislexia; trastorno específico de lectura; inequidad; socioeconómica; rendimiento académico.

\begin{abstract}
The relationship between reader performance and academic performance has been described and the impact on both of them of the child's socioeconomic vulnerability. On the other hand, there is no abundance of literature on the impact of socioeconomic status on the presence of developmental dyslexia. On a sample
\end{abstract}


of 216 Primary school children from public and private schools in Panama City, with 12 participants with dyslexia, the variables of reader performance, socioeconomic category, and academic performance were studied. However, no direct link was found in this study between the participants' socioeconomic level and the prevalence of dyslexia. Possibly the group located in the lowest part of the scale has tripled its probabilities of presenting the disorder compared to the rest of the sample. The results indicate a more linear relationship between socioeconomic level and reading performance and the negative impact that dyslexia and low reading competition have on academic performance. It is proposed as the hypothesis that the results could be consistent with the presence in a dyslexic disorder of a neurobiological and genetic component, but whose impact could be modulated in intensity by the environmental component and a reflection is made on the low school performance serving that could be avoided with active policies to address dyslexia.

Keywords: Dyslexia; specific reading impairment; inequity; socioeconomic; academic performance.

\section{Introducción}

Según la clasificación CIE-11 (Organización Mundial de la Salud, 2018), el trastorno del desarrollo del aprendizaje con dificultades en la lectura (código 6A03.0), habitualmente denominado también dislexia del desarrollo (DD), se caracteriza por dificultades significativas y persistentes en el aprendizaje de habilidades académicas relacionadas con la lectura, como precisión en la lectura de la palabra, fluidez en la lectura y comprensión de la lectura. Su carácter neurobiológico (Soriano-Ferrer y Piedra Martínez, 2017) y, al menos parcialmente, genético (De la Peña y Bernabéu, 2018) se consideran probados. Para su detección es necesario determinar una alteración significativa y crónica de la competencia lectora en ausencia de daño sensorial, trastorno intelectual, trastornos emocionales primarios o falta de instrucción específica (Manual DSM-V de la Asociación Americana de Psiquiatría, 2013).

No existen estudios sobre las consecuencias de la DD en Panamá, aunque sí se dispone de estudios descriptivos de la competencia lectora de los jóvenes panameños. Esta se caracteriza por un muy bajo nivel, puesto que, según los resultados de las pruebas CRECER de 2016, un 83\% de los participantes se situó por debajo de lo que se consideró un rendimiento mínimo en comprensión lectora (Paredes, 2017) y que en el informe del Programa Internacional para la Evaluación de Estudiantes (PISA) de 2018 el país ocupó en la posición 71 (de 77 participantes) en cuanto a desempeño lector (MEDUCA y OCDE, 2019).

Por otra parte, tampoco se encuentran publicaciones que traten de revelar una posible relación entre la DD o el nivel de rendimiento lector en Panamá y dos problemas relevantes para el país: el fracaso escolar y la inequidad socioeconómica. Los niveles de fracaso y abandono escolar son elevados (Gordón, 2019) y se trata de uno de los países más desiguales de América Latina (Astudillo, Fernández y Garcimartín, 2019).

El rendimiento lector insuficiente se ha revelado en la literatura científica como una de las causas conocidas del fracaso escolar, fenómeno sin duda multicausal para cuyo estudio hay que tener además en cuenta factores socioeconómicos, nutricionales, pedagógicos, familiares, psicolingüísticos y sistémicos (Rosario, 2016). En la misma línea, la DD, como el más prevalente de los trastornos de aprendizaje, también es específicamente reconocida como una de las principales causas de fracaso escolar, dado que los estudiantes que la padecen tienden a ver progresivamente acrecentado su retraso lector y académico con respecto a sus compañeros (Francis, Stuebing, Shaywitz, Shaywitz, y Fletcher, 1996). El reconocimiento del impacto de este trastorno sobre el desempeño académico ha llevado a muchos países a incluir en su normativa educativa adaptaciones escolares y curriculares para permitir un correcto de desarrollo de los estudiantes que lo padecen (Barba, Suárez, Jomarrón y Navas, 2019).

El impacto de la variable socioeconómica, con la que se relaciona la inequidad, sobre la prevalencia de trastornos de la lectura no ha sido profusamente estudiada. Por el contrario, sí ha sido bien descrita su influencia sobre el desarrollo lector (Catts, Fey, Zhang y Tomblin, 2001; National Assessment of Educational Progress, 
1991). En la región Latinoamericana, existe abundancia de estudios al respecto, encontrando siempre efectos significativos de la deprivación socioeconómica sobre el desarrollo lector (Canales Gabriel, 2014; Cuadro y Balbi, 2012; Cueto, León y Muñoz, 2016; Dioses, Evangelista, Basurto, Morales y Alcántara, 2010; Hernández Padilla y Bazán Ramírez, 2016; Infante, 2006; Marco y Saz, 2006; Urquijo, Coni, y Fernandes, 2015). De hecho, la influencia de la inequidad no solo se extiende a la decodificación y al acceso al sentido, sino que se remonta a los propios precursores psicolingüísticos de la lectura, las capacidades cognitivas de instalación previa sobre las que se asienta el aprendizaje lector, como la conciencia fonológica o el léxico, cuyo nivel se ve afectado en niños/as que evolucionan en entornos más vulnerables (Espinoza y Rosas, 2019; Flórez-Romero y AriasVelandia, 2010; Ortiz Padilla, Becerra, Vega, Sierra y Cassiani, 2010).

\section{Método}

\section{Objetivo}

El presente trabajo trata de describir la posible relación existente entre el rendimiento lector o su trastorno específico, la dislexia, y variables como el desempeño escolar y la inequidad socioeconómica en una muestra de estudiantes panameños con DD y con rendimiento lector dentro de la normalidad, mediante un diseño no experimental, transversal, no probabilístico y descriptivo de corte cuantitativo.

\section{Población y muestra}

Se partió de una muestra intencional inicial de 216 estudiantes de $4^{\circ}(n=74), 5^{\circ}(n=72)$ y $6^{\circ}(n=70)$ grado de Educación Primaria de 6 escuelas de la Ciudad de Panamá. La muestra fue pareada por sexo y se compuso de estudiantes del sistema escolar público $(n=139)$ y del privado, o particular $(n=77)$. El cumplimiento estricto de los principios de la Declaración de Helsinki en cuanto a respeto de la integridad, anonimato de los participantes y obtención de consentimiento y asentimiento informado fue supervisado por un comité de bioética.

\section{Instrumentos}

Se emplearon las subpruebas de Lectura de Palabras, Lectura de Pseudopalabras y comprensión lectora de la prueba PROLEC-R (Cuetos, Rodríguez, Ruano y Arribas, 2014), empleando la baremación efectuada con población de la región metropolitana de Lima (Cayhualla, Chilón, \& Espíritu, 2011, 2013), para determinar qué participantes se encontraban en dificultad lectora severa (-2 desviaciones estándar en alguna de las pruebas de decodificación o en la comprensión lectora, presentando en este último caso simultáneamente al menos -1 desviación estándar en las dos subpruebas de decodificación). Se utilizó la prueba WISC-IV (Wechsler, 2007) para la medición del cociente intelectual, con el fin de descartar como casos a aquellos participantes que, aun teniendo una desviación significativa en lectura, tuvieran un rendimiento intelectual que pudiera justificar la dificultad en el aprendizaje de la lectura.

\section{Procedimiento de recogida y análisis de datos}

Los estudiantes que cumplían los criterios diagnósticos de dificultad significativa de la lectura en ausencia de causa intelectual (cociente intelectual superior a 70) fueron detectados como se detalla en el apartado anterior. Se comprobó que no existiera daño sensorial severo que pudiera explicar el trastorno de lectura. 
Las 6 escuelas a las que pertenecían los participantes fueron distribuidas en 4 niveles socioeconómicos categóricos ordinales, siguiendo los siguientes criterios: gastos de escolaridad, recursos tecnológicos y acceso a internet, recursos de aprendizaje, presencia de atención docente especializada y ratios de alumnos por grupo. Finalmente, la categorización de la muestra del estudio quedó organizada como se muestra en la figura 1.

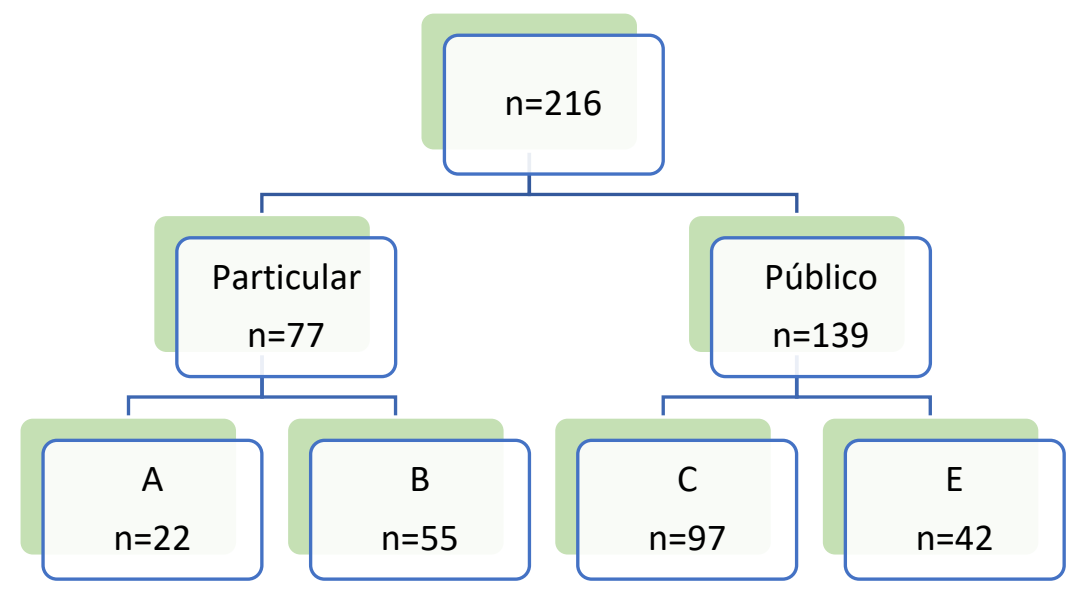

Figura 1. Distribución de la muestra.

Nota: Categorías, A= Escuela particular, nivel socioeconómico alto; $\mathrm{B}=$ Escuela particular, nivel socioeconómico medio; C= Escuela pública, nivel socioeconómico medio; $\mathrm{D}=$ Escuela pública, nivel socioeconómico bajo

No se dispuso para la construcción de la muestra de ninguna escuela de la categoría D (nivel socioeconómico medio-bajo), por lo que la categoría E queda marcadamente diferenciada de las tres primeras. No se identificaron en la zona metropolitana escuelas públicas de nivel socioeconómico alto, ni escuelas particulares de nivel socioeconómico bajo para diseñar categorías con estos rasgos.

Se evaluó individualmente el rendimiento lector de cada uno de los participantes, siguiendo el Modelo de la Doble Ruta (Coltheart y Rastle, 1994): concretamente, se evaluó el uso de la ruta léxica de lectura midiendo precisión y velocidad de la lectura de palabras y el de la ruta fonológica de lectura midiendo precisión y velocidad de la lectura de pseudopalabras. En ambos casos, se empleó un índice combinado de precisión y velocidad. Se evaluó también la eficacia lectora con la subprueba de comprensión lectora. Sobre la muestra de 216 estudiantes se detectaron, siguiendo un criterio diagnóstico estricto ( -2 desviaciones estándar en rendimiento lector), 12 participantes con perfil de trastorno específico del aprendizaje de la lectura, tras descartar a un estudiante con cociente intelectual inferior a 70 (para un análisis más detallado, ver CubillaBonnetier, Solís-Rodríguez, Farnum y Caballero, 2020).

Paralelamente, se obtuvo información sobre el promedio académico de los estudiantes, distribuyéndolos en 3 grupos categóricos ordinales:

1: Promedio académico entre 4.5 y 5.

2: Promedio académico entre 4.4 y 4.

3: Promedio académico igual o inferior a 3.9.

Para la extracción de los resultados del estudio, se procedió a comparar promedios de rendimiento lector y de prevalencia de dislexia entre los participantes por grupos de nivel socioeconómico y de promedio académico.

Para poder destacar determinados resultados, cuando procedió se emplearon para el análisis las herramientas habitualmente usadas en salud pública de Riesgo Relativo (RR), o Razón de Riesgos (Fajardo- 
Gutiérrez, 2017), que pone en evidencia el aumento del riesgo de presentar una condición por pertenecer a un grupo con un determinado factor, y la de Proporción de Riesgo Atribuible en el grupo expuesto (RA $\mathrm{exp}_{\%}$ ), o fracción etiológica, que calcula la proporción de casos expuestos a un factor o condición que podrían evitar presentar una patología o encontrarse en determinada situación si se evitara su exposición al factor (MorenoAltamirano, López-Moreno y Corcho-Berdugo, 2000).

\section{Resultados}

\section{Dislexia e inequidad}

Tabla 1. Distribución de la prevalencia de DD, por tipo de escuela según nivel socioeconómico.

\begin{tabular}{cccc}
\hline $\begin{array}{c}\text { Nivel socioeconómico } \\
\text { de la escuela }\end{array}$ & $\begin{array}{c}\text { Frecuencia de } \\
\text { participantes con DD }\end{array}$ & $\mathbf{n}$ & Prevalencia de DD \\
\hline A & 2 & 22 & $9.09 \%$ \\
B & 2 & 55 & $3.64 \%$ \\
C & 3 & 97 & $3.09 \%$ \\
E & 5 & 42 & $11.90 \%$ \\
Total & $\mathbf{1 2}$ & $\mathbf{2 1 6}$ & $\mathbf{5 . 5 6 \%}$ \\
\hline
\end{tabular}

La prevalencia encontrada en el grupo de estudiantes de escuelas públicas $(n=139)$ fue de $5.19 \%$ frente a $5.76 \%$ en el grupo de participantes de escolarizados en el sistema particular $(n=77)$. Esta diferencia no resultó estadísticamente significativa $(\mathrm{p} \leq 0.05)$.

Se empleó la herramienta del riesgo relativo (RR), con la fórmula:

$$
\mathrm{RR}=\frac{\text { Riesgo del grupo expuesto al factor }}{\text { Riesgo del grupo no expuesto al factor }}
$$

En este caso, se calculó el riesgo relativo de presentar dislexia estando en la escuela del grupo E:

$$
\mathrm{RR}=\frac{\frac{5}{42}}{\frac{7}{174}}=2.96
$$

Este resultado indica que los participantes del grupo socioeconómico E (escuela pública de nivel socioeconómico bajo ven multiplicado su riesgo de presentar DD por 2.96).

\section{Dislexia y desempeño escolar}

Tabla 2. Distribución de los participantes con riesgo de dislexia, según su escala de rendimiento académico

\begin{tabular}{ccccc}
\hline $\begin{array}{c}\text { Grupo por } \\
\text { calificación } \\
\text { académica }\end{array}$ & $\begin{array}{c}\text { Frecuencia } \\
\text { absoluta de } \\
\text { participantes }\end{array}$ & $\begin{array}{c}\text { Frecuencia } \\
\text { relativa de } \\
\text { participantes }\end{array}$ & $\begin{array}{c}\text { Frecuencia } \\
\text { absoluta de } \\
\text { participantes } \\
\text { con DD }\end{array}$ & $\begin{array}{c}\text { Prevalencia de } \\
\text { DD en el } \\
\text { grupo }\end{array}$ \\
\hline 1 & 43 & $19.91 \%$ & 1 & $2.33 \%$ \\
2 & 102 & $47.22 \%$ & 4 & $3.92 \%$ \\
3 & 71 & $32.87 \%$ & 7 & $9.86 \%$ \\
Total & $\mathbf{2 1 6}$ & $\mathbf{1 0 0 \%}$ & $\mathbf{1 2}$ & $\mathbf{5 . 5 6 \%}$ \\
\hline
\end{tabular}


La proporción en que aumenta el riesgo de encontrarse en grupo 3 (aproximadamente el tercio de la muestra con peor rendimiento académico) al tener DD, será:

$$
\mathrm{RR}=\frac{\frac{7}{12}}{\frac{64}{204}}=\mathbf{1 . 8 6}
$$

En este caso, puede ser ilustrativo llevar a cabo el cálculo de la proporción de riesgo atribuible en el grupo expuesto $\left(\mathrm{RA}_{\exp } \%\right)$, que en este caso significaría la proporción de casos con $\mathrm{DD}$ en el grupo 3 de promedio académico que podrían evitar verse en este grupo si se eliminara su DD, mediante la fórmula:

$$
\mathrm{RA}_{\mathrm{exp}} \%=\frac{\text { prevalencia de } F E \text { entre casos con DD-prevalencia de FE en el resto }}{\text { prevalencia de FE entre casos con DD }} \times 100
$$

Concretamente:

$$
\mathrm{RA}_{\exp } \%=\frac{\frac{7}{12}-\frac{64}{204}}{\frac{7}{12}} x 100=\mathbf{4 6 . 2 2}
$$

Esto significa que de cada 100 casos de estudiantes con DD situados en el tercio con peor rendimiento académico, 46 de ellos no se encontrarían en dicha situación escolar de no presentar DD.

\section{Rendimiento lector e inequidad}

Tabla 3. Promedios de los diferenciales individuales* en los Índices Generales ${ }^{1}$ de las pruebas del PROLEC$R$ relacionadas con el rendimiento lector, por grupos (variable socioeconómica)

\begin{tabular}{cccc}
\hline $\begin{array}{c}\text { Nivel } \\
\text { socioeconómico } \\
\text { de la escuela }\end{array}$ & $\begin{array}{c}\text { Promedio de } \\
\text { diferenciales } \\
\text { individuales en lectura } \\
\text { de palabras }\end{array}$ & $\begin{array}{c}\text { Promedio de diferenciales } \\
\text { individuales en lectura de } \\
\text { pseudopalabras }\end{array}$ & $\begin{array}{c}\text { Promedio de } \\
\text { diferenciales } \\
\text { individuales en } \\
\text { comprensión de } \\
\text { textos }\end{array}$ \\
\hline A $(n=22)$ & 12,15 & 5,44 & 0,74 \\
B $(n=55)$ & $-2,88$ & $-0,76$ & 0,33 \\
C $(n=97)$ & 6,26 & 2,66 & 0,23 \\
E $(n=42)$ & $-17,05$ & $-8,00$ & $-1,36$ \\
\hline
\end{tabular}

*Con el fin de controlar el efecto de las diferencias entre grados escolares, se calculó para cada individuo la diferencia entre su resultado en cada una de las tareas y el promedio de puntuaciones de los participantes de su mismo grado en el conjunto de la muestra.

Como se puede observar, la progresividad de la reducción del rendimiento lector a medida que se desciende de estrato socioeconómico solo se ve alterada entre los dos grupos de nivel socioeconómico medio, B y $\mathrm{C}$, donde encontramos una ventaja del grupo $\mathrm{C}$ de estudiantes de escuelas públicas de clase media en las tareas de decodificación (lectura de palabras y pseudopalabras).

\footnotetext{
${ }^{1}$ Los índices generales son puntuaciones que combinan precisión y velocidad en cada una de las subpruebas de la prueba PROLEC-R 
Tabla 4. Promedio de los diferenciales individuales en los Índices Generales en las pruebas del PROLEC-R relacionadas con el rendimiento lector, por tipo de escuela (pública/particular)

\begin{tabular}{cccc}
\hline Tipo de escuela & $\begin{array}{c}\text { Promedio de } \\
\text { diferenciales } \\
\text { individuales en } \\
\text { lectura de } \\
\text { palabras }\end{array}$ & $\begin{array}{c}\text { Promedio de } \\
\text { diferenciales } \\
\text { individuales en } \\
\text { lectura de } \\
\text { pseudopalabras }\end{array}$ & $\begin{array}{c}\text { Promedio de } \\
\text { diferenciales } \\
\text { individuales en } \\
\text { comprensión de } \\
\text { textos }\end{array}$ \\
\hline Pública $(\mathrm{n}=139)$ & $-0,78$ & $-0,56$ & $-0,25$ \\
Particular $(\mathrm{n}=77)$ & 1,41 & 1,01 & 0,45 \\
\hline
\end{tabular}

Aunque aparecen diferencias en todas las tareas de rendimiento lector en favor del grupo de participantes de escuelas particulares, dichas diferencias no resultaron estadísticamente significativas en base a la prueba $\mathrm{T}$ de Student $(\mathrm{p} \leq 0.05)$ en ninguna de las tres tareas: lectura de palabras $(\mathrm{t}=-0.505 ; \mathrm{p}=0.614)$, lectura de pseudopalabras $(\mathrm{t}=-0.603 ; \mathrm{p}=0.547)$ y comprensión de textos $(\mathrm{t}=-1.784 ; \mathrm{p}=0.076)$.

\section{Rendimiento lector y desempeño escolar}

Tabla 5. Promedio de los resultados en los Índices Generales en las pruebas del PROLEC-R por grado y calificación académica

\begin{tabular}{ccccc}
\hline Grado & $\begin{array}{c}\text { Grupo por } \\
\text { calificación } \\
\text { académica }\end{array}$ & $\begin{array}{c}\text { Promedio en } \\
\text { lectura de palabras }\end{array}$ & $\begin{array}{c}\text { Promedio en lectura de } \\
\text { pseudopalabras }\end{array}$ & $\begin{array}{c}\text { Promedio en } \\
\text { comprensión } \\
\text { de textos }\end{array}$ \\
\hline \multirow{2}{*}{$\mathbf{4}^{\circ}$} & $1(\mathrm{n}=16)$ & 72.24 & 51.54 & 11.88 \\
& $2(\mathrm{n}=33)$ & 83.82 & 53.73 & 13.38 \\
& $3(\mathrm{n}=25)$ & 59.94 & 39.14 & 10.56 \\
\hline \multirow{2}{*}{$\mathbf{5}^{\circ}$} & $1(\mathrm{n}=19)$ & 84.30 & 57.99 & 13.47 \\
& $2(\mathrm{n}=35)$ & 86.22 & 53.45 & 13.09 \\
& $3(\mathrm{n}=18)$ & 74.36 & 44.91 & 12.89 \\
\hline \multirow{2}{*}{$\mathbf{6}^{\circ}$} & $1(\mathrm{n}=8)$ & 105.89 & 64.03 & 14.50 \\
& $2(\mathrm{n}=34)$ & 103.71 & 67.18 & 13.06 \\
& $3(\mathrm{n}=28)$ & 91.55 & 59.65 & 13.21 \\
\hline
\end{tabular}

En el seno de cada uno de los 3 grados, se seleccionó en cada una de las 3 tareas de lectura a todos los estudiantes con puntajes situados al menos una desviación estándar por debajo del promedio del grado (estudiantes con dificultades moderadas o severas en la tarea). Se procedió a calcular el Riesgo Relativo de tener un promedio académico del grupo 3 (del peor tercio) teniendo dificultades en cada una de las tareas.

Tabla 6. Riesgo relativo de tener un promedio académico del grupo 3, teniendo un rendimiento al menos 1 desviación estándar en cada una de las tareas, por grado académico

\begin{tabular}{cccc}
\hline Grado & $\begin{array}{c}\text { Lectura de } \\
\text { palabras }\end{array}$ & $\begin{array}{c}\text { Lectura de } \\
\text { pseudopalabras }\end{array}$ & $\begin{array}{c}\text { Promedio en } \\
\text { comprensión } \\
\text { de textos }\end{array}$ \\
\hline $\mathbf{4}^{\circ}$ & $\mathrm{RR}=3.02$ & $\mathrm{RR}=2.76$ & $\mathrm{RR}=3.03$ \\
$\mathbf{5}^{\circ}$ & $\mathrm{RR}=1.59$ & $\mathrm{RR}=1.59$ & $\mathrm{RR}=1.77$ \\
$\mathbf{6}^{\circ}$ & $\mathrm{RR}=1.61$ & $\mathrm{RR}=1.3$ & $\mathrm{RR}=1.17$ \\
\hline
\end{tabular}


Como ejemplo para su interpretación, un estudiante de $4^{\circ}$ grado cuya competencia en la prueba de lectura de palabras se encuentre por debajo del promedio de su grado en, al menos, una desviación estándar multiplicará por 3.02 sus probabilidades de tener un promedio académico del grupo 3.

\section{Discusión y conclusiones}

Los resultados no permiten afirmar que la inequidad aumente el riesgo de presentar dislexia, ya que los promedios de prevalencia no aumentaron de forma progresiva a medida que se descendía el nivel socioeconómico en los grupos categóricos ordinales creados para dicho efecto. Fue el grupo de mayor nivel socioeconómico el que alteró la tendencia progresiva, puesto que presentó una notable prevalencia de 9,09\%. No obstante, a la hora de valorar los resultados, hay que tomar en cuenta el reducido tamaño de la muestra del grupo A $(n=22)$ y del número de casos encontrados en él (2), pues esta prevalencia podría estar parcialmente asociada al azar. Merece atención la alta prevalencia de DD en el grupo $\mathrm{E}(\mathrm{n}=42), 11.9 \%$, con participantes especialmente sujetos a la inequidad por su ubicación en una zona de la ciudad con altos niveles de vulnerabilidad social y económica. De hecho, los resultados parecen indicar que pertenecer a dicho grupo de la muestra prácticamente triplica el riesgo de presentar dislexia.

El hecho de que la variable socioeconómica no se relacione inversamente con la de prevalencia de dislexia es coherente con el carácter neurobiológico, y en parte genético, del trastorno, que podría verse como una especie de "lotería" por la probada predisposición familiar poligénica (Preilowski y Matute, 2011). No obstante, la reducción de la capacidad lectora que causa la DD puede verse afectada positiva o negativamente por el volumen y la calidad de la estimulación psicolingüística recibida por el sujeto, el acompañamiento familiar en actividades de lectura y el adiestramiento escolar recibido, lo cual puede ayudar a interpretar la muy elevada prevalencia encontrada en el grupo E, a la luz de los bajos niveles de rendimiento lector que presenta el conjunto del grupo, que se contraponen a los del grupo A, el otro grupo en el que la prevalencia de DD fue alta. De esta forma, se combinarían los factores estructurales y los ambientales para la determinación de la severidad del trastorno: una estimulación adecuada e incluso una intervención específica podrían elevar el rendimiento lector de niños con perfil neurobiológico de DD y permitirles así superar el punto de corte de 2 desviaciones estándar por debajo del promedio en rendimiento lector, teniendo en cuenta el carácter continuo de la distribución de la variable de capacidad lectora en la población (Artigas-Pallarés, 2009). No obstante, también podría darse el fenómeno inverso y que perfiles inicialmente menos severos pudieran caer por debajo del punto de corte por una estimulación inadecuada.

En cuanto a la influencia de la dislexia sobre el rendimiento escolar, parece existir una relación entre ambas variables, puesto que cuando descendemos en los tres niveles de rendimiento medido por el promedio académico aumenta la prevalencia de dislexia, aunque por el diseño del estudio no se puede afirmar que dicha relación sea causal. Los niños y niñas con dislexia multiplican por 1.86 veces su riesgo de encontrarse en el peor tercio de la muestra en cuanto a promedio académico con respecto a los normo-lectores. Esto parece confirmar al trastorno específico del aprendizaje de la lectura como un factor de riesgo para el fracaso escolar (en la Ciudad de Panamá, prácticamente duplica dicho riesgo). Aunque no se han encontrado estudios que cuantifiquen la porción de fracaso escolar atribuible a la DD, la existencia de esta relación es coherente con la sintomatología de la dislexia, que se caracteriza por problemas de exactitud y de fluidez en la lectura (Snowling y Hulme, 2012) que a su vez pueden provocar dificultades de comprensión lectora (Álvarez-Cañizo, SuárezCoalla, y Cuetos, 2015) y el exceso de carga cognitiva (Martin, Kronbichler, y Richlan, 2016) que puede provocar la falta de automatización de la lectura.

Entre los participantes con DD que se encuentran en el grupo C de desempeño académico, se calculó que el $46 \%$ de ellos no se encontrarían en esta situación de no ser por la presencia del trastorno. Este dato, aunque preocupante, es también una invitación al abordaje decidido de la DD, puesto que con la prevalencia encontrada en el estudio (5.56\%), entre la población de estudiantes panameños de Primaria, Premedia y Media 
encontraríamos 44'701 casos positivos, en base a los datos disponibles de población escolar ${ }^{2}$ (Ministerio de Educación de Panamá, 2019). Con la proporción de riesgo atribuible encontrada, si se llevaran a cabo políticas universales de salud y educación para la compensación de los trastornos específicos del aprendizaje de la lectura, se podrían evitar 20'661 promedios académicos bajos, directamente relacionados con el fracaso escolar, puesto que es sabido que una intervención intensiva basada en evidencia puede proveer al niño/a afectado/a de mecanismos neurológicos de lectura funcionales (Simos et al., 2002). Al tratarse de un trastorno crónico que se extiende a la adolescencia y a la edad adulta (Undheim, 2009), podemos extrapolar el enorme impacto que tendría también la puesta en marcha de programas de detección masiva en la calidad de vida de la población adulta futura.

Los hallazgos sugieren que la variable socioeconómica sí parece producir un impacto claro sobre el rendimiento lector. Este efecto aparece descrito en la literatura (Molfese et al., 2003) y particularmente en los estudios producidos en el continente (Urquijo et al., 2015, entre otros ya mencionados anteriormente).

La diferencia en la estimulación recibida por los participantes del grupo de nivel socioeconómico E no parece deberse a su pertenencia al sistema escolar público, puesto que la prevalencia de DD entre los niños y niñas escolarizados en este sistema en el conjunto de la muestra no resultó superior a la de los escolarizados en el sistema particular y que el rendimiento lector de los participantes de escuelas públicas no resultó estadísticamente inferior al de los de escuelas privadas, aunque este último resultado sea contradictorio con el ofrecido por el estudio PISA de 2018 (MEDUCA y OCDE, 2019), que, con un tamaño muestral mucho mayor, encontró un diferencial muy significativo de rendimiento lector entre los centros públicos y los privados del país, en favor de los últimos. Esto podría sugerir que procede buscar, mediante un estudio específico, las causas de la fuerte caída en el rendimiento lector y del aumento de casos detectados con DD en el grupo E en la estimulación lingüística y metalingüística recibida en el hogar, así como en el modelado familiar temprano de conductas lectoras, puesto que se trata de factores reconocidos como predictores del éxito en el aprendizaje lector (Gallego, 2006).

La tabla $\mathrm{n}^{\circ} 5$ muestra que el rendimiento lector no parece marcar la diferencia entre estudiantes con buenos resultados y estudiantes del grupo intermedio en cuanto a promedio académico. En cambio, el grupo de estudiantes situados en el tercio inferior en cuanto a desempeño académico presenta en todos los grados un rendimiento lector sensiblemente inferior al del resto de estudiantes en tareas de decodificación (lectura de palabras y pseudopalabras). Sin embargo, sorprendentemente, las diferencias de este grupo con los demás no resultaron significativas en el ámbito de la eficacia lectora (comprensión de textos), salvo en $4^{\circ}$ grado. Generalmente, se observa (tabla $\mathrm{n}^{\circ}$ ) que la falta de competencia lectora aumenta el riesgo relativo de encontrarse en el tercio de la muestra con peores rendimientos y, aunque este efecto es fuerte en $4^{\circ}$ grado, parece ir debilitándose a medida que se avanza hacia el final de la Educación Primaria.

Para proseguir con el estudio del fenómeno del bajo rendimiento lector en el país y de sus causas para un mejor abordaje, se sugiere la puesta en marcha de un estudio con una muestra de mayor tamaño y de tipo probabilístico que introduzca nuevas posibles variables independientes causales de los fracasos lector y escolar y con medios que permitan una evaluación cuantitativa directa de la variable socioeconómica (por ejemplo, mediante encuestas estructuradas a las familias) y del rendimiento académico (obteniendo promedios generales, pero también detallados por asignaturas).

A modo de conclusión, los resultados muestran que, aunque no se puede afirmar que la inequidad aumente las tasas de dislexia, encontramos una muy alta prevalencia entre los participantes de nivel socioeconómico más bajo. Tal vez influya en este fenómeno la relación directa que sí aparece, en la línea del conocimiento existente, entre la variable socioeconómica y la de rendimiento lector. Aparece igualmente un efecto nocivo de la dislexia $\mathrm{y}$, de forma general, de los bajos niveles de lectura sobre el desempeño académico, de manera que normalizando la capacidad lectora de los niños y niñas se podría evitar una porción significativa del fracaso escolar en el país: al menos en 20’000 niños y niñas con bajos promedios académicos con una detección e intervención

\footnotetext{
${ }^{2}$ Con un total de 803'983 estudiantes en 2019.
} 
sistemáticas de los casos de trastorno de la lectura y otra porción sin estimar de rendimientos limitados por la escasa funcionalidad de la lectoescritura, sin que se trate forzosamente de casos de DD.

\section{Financiación}

El presente trabajo fue financiación por la Universidad Católica Santa María la Antigua USMA de Panamá, Proyecto SRUI-CPEI-ID-2018-2019-009.

\section{Referencias}

Álvarez-Cañizo, M., Suárez-Coalla, P., \& Cuetos, F. (2015). The role of reading fluency in children's text comprehension. Frontiers in Psychology, 6(NOV). https://doi.org/10.3389/fpsyg.2015.01810

American Psychiatric Association, A. (2013). Diagnostic and statistical manual of mental disorders, (DSM-V). Arlington, VA: American Psychiatric Publishing.

Artigas-Pallarés, J. (2009). Dislexia: enfermedad, trastorno o algo distinto. Revista de Neurología, 48(2), $63-$ 69.

Astudillo, J., Fernández, M., \& Garcimartín, C. (2019). La desigualdad de Panamá: Su carácter territorial y el papel de las inversiones públicas. https://doi.org/10.18235/0001776

Barba, M. N., Suárez, N., Jomarrón, L., \& Navas, C. R. (2019). Tendencias actuales de la investigación en dislexia y necesidad de formación docente. Revista Cubana de Medicina Militar, 48(supl. 1).

Canales Gabriel, R. (2014). Asociación entre factores neuropsicológicos, procesos cognitivos y niveles de lectura en niños de diferente nivel socioeconómico del Callao. Revista de Investigación En Psicología, 16(2), 89. https://doi.org/10.15381/rinvp.v16i2.6548

Catts, H. W., Fey, M. E., Zhang, X., \& Tomblin, J. B. (2001). Estimating the risk of future reading difficulties in kindergarten children: A research-based model and its clinical implementation. Language, Speech, and Hearing Services in Schools, 32(1), 38-50. https://doi.org/10.1044/0161-1461(2001/004)

Cayhualla, N., Chilón, D., \& Espíritu, R. (2011). Adaptación de la Batería de Evaluación de los Procesos Lectores Revisada PROLEC-R en estudiantes de Primaria de Lima Metropolitana (Pontificia $\begin{array}{lllll}\text { Universidad Católica } & \text { del } & \text { Perú). } & \text { Retrieved }\end{array}$ http://tesis.pucp.edu.pe/repositorio/handle/20.500.12404/1309?show=full

Cayhualla, N., Chilón, D., \& Espíritu, R. (2013). Adaptación psicométrica de la Batería de Evaluación de los Procesos Lectores Revisada (PROLEC-R). Propósitos y Representaciones, 1(1), 39-57. https://doi.org/10.20511/pyr2013.v1n1.3

Coltheart, M., \& Rastle, K. (1994). Serial Processing in Reading Aloud : Evidence for Dual-Route Models of Reading. Journal of Experimental Psychology: Human Perception and Performance, 20(6), 1197-1211.

Cuadro, A., \& Balbi, A. (2012). Las diferencias socioeconómicas y la lectura: claves para analizar los resultados de las evaluaciones PISA. Revista Neuropsicología Latinoamericana, 4(1), 57-64.

Cubilla-Bonnetier, D., Solís-Rodríguez, A., Farnum, F., \& Caballero, J. (2020). Prevalencia de dislexia del desarrollo en estudiantes panameños. Investigación y Pensamiento Crítico.

Cueto, S., León, J., \& Muñoz, I. G. (2016). Conductas, estrategias y rendimiento en lectura en PISA: análisis 
para el Perú. REICE. Revista Iberoamericana Sobre Calidad, Eficacia y Cambio En Educación, 14(3), 5-32. Retrieved from https://dialnet.unirioja.es/servlet/articulo?codigo=5536818

Cuetos, F., Rodríguez, B., Ruano, E., \& Arribas, D. (2014). Test PROLEC-R. Batería de Evaluación de los Procesos Lectores, revisada (5a edición). (TEA Edicio). Madrid: TEA Ediciones.

De la Peña, C., \& Bernabéu, E. (2018). Dislexia y discalculia: una revisión sistemática actual desde la neurogenética. Universitas Psychologica, 17(3), 1-11.

Dioses, A., Evangelista, C., Basurto, A., Morales, M., \& Alcántara, M. (2010). Procesos cognitivos implicados en la lectura y escritura de niños y niñas del tercer grado de educación primaria residentes en Lima y Piura. Revista de Investigación En Psicología, 13(1), 13-40.

Espinoza, V., \& Rosas, R. (2019). Diferencias iniciales en el proceso de acceso al lenguaje escrito según nivel socioeconómico. Perspectiva Educacional, 58(3), 23-45. https://doi.org/10.4151/07189729-vol.58-iss.3art.955

Fajardo-Gutiérrez, A. (2017). Medición en epidemiología: prevalencia, incidencia, riesgo, medidas de impacto. Revista Alergia Mexico, 64(1), 109-120. https://doi.org/10.29262/ram.v64i1.252

Flórez-Romero, R., \& Arias-Velandia, N. (2010). Evaluación de conocimientos previos del aprendizaje inicial de lectura. Magis, 2(4), 329-343.

Francis, D. J., Stuebing, K. K., Shaywitz, S. E., Shaywitz, B. A., \& Fletcher, J. M. (1996). Developmental Lag Versus Deficit Models of Reading Disability: A Longitudinal, Individual Growth Curves Analysis. Journal of Educational Psychology, 88(1), 3-17. https://doi.org/10.1037/0022-0663.88.1.3

Gallego, C. (2006). Los prerrequisitos lectores. Morelia (México). Congreso Internacional de Lectoescritura. Retrieved from http://www.waece.org/cd_morelia2006/ponencias/gallego.htm

Gordón, F. (2019). Mayor índice de fracaso escolar se mantiene en Matemáticas y Español | Panamá América. Panamá América. Retrieved from https://www.panamaamerica.com.pa/sociedad/mayor-indice-defracaso-escolar-se-mantiene-en-matematicas-y-espanol-1152566

Hernández Padilla, E., \& Bazán Ramírez, A. (2016). Efectos contextuales, socioeconómicos y culturales, sobre los resultados de México en Lectura en PISA 2009. REICE. Revista Iberoamericana Sobre Calidad, Eficacia y Cambio En Educación, 14.2(2016). https://doi.org/10.15366/reice2016.14.2.005

Infante, M. (2006). Las dificultades específicas de aprendizaje en lectura y su relación con habilidades lingüísticas en contextos socioeconómicos diversos. Revista Chilena de Fonoaudiología, 7(1), 29-44. https://doi.org/10.5354/0719-4692.2006.48360

Marco, M. A., \& Saz, A. (2006). Influencia del nivel socioeconómico y cultural en el rendimiento de los estudiantes de Tercero Básico y Graduandos del año 2006. Retrieved from http://www.mineduc.gob.gt/digeduca/documents/papers/paper_nivel socioeconomico paper.pdf

Martin, A., Kronbichler, M., \& Richlan, F. (2016). Dyslexic brain activation abnormalities in deep and shallow orthographies: A meta-analysis of 28 functional neuroimaging studies. Human Brain Mapping, 37(7), 2676-2699. https://doi.org/10.1002/hbm.23202

MEDUCA (Ministerio de Educación de Panamá). (2019). Boletín de Estadística Educativa. Retrieved from http://www.meduca.gob.pa/direccion-plane/estadisticas

MEDUCA (Ministerio de Educación de Panamá), \& OCDE (Organización para la Cooperación y el Desarrollo Económicos). (2019). Informe de resultados del Programa para la Evaluación Internacional de Estudiantes

(PISA).

Retrieved

from 
http://www.meduca.gob.pa/sites/default/files/WEB/pisa/PISA2018_Panamá_ResumenEjecutivo_(1)compressed.pdf

Molfese, V. J., Modglin, A., \& Molfese, D. L. (2003). The role of environment in the development of reading skills: a longitudinal study of preschool and school-age measures. Journal of Learning Disabilities, 36(1), 59-67. https://doi.org/10.1177/00222194030360010701

Moreno-Altamirano, A., López-Moreno, S., \& Corcho-Berdugo, A. (2000). Principales medidas en epidemiología. Revista de Salud Pública de México, 42(4), 337-348.

National Assessment of Educational Progress. (1991). The 1989-90 national assessment of reading and literature. Denver, CO.: Autor.

Organización Mundial de la Salud, O. (2018). Clasificación Internacional de Enfermedades undécima revisión (CIE-11). Ginebra: Organización Mundial de la Salud, OMS.

Ortiz Padilla, M., Becerra, J., Vega, K., Sierra, P., \& Cassiani, Y. (2010). Madurez para la lectoescritura en niños/as de instituciones con diferentes estratos socioeconómicos. Psicogente, 13(23), 107-130. Retrieved from https://dialnet.unirioja.es/servlet/articulo?codigo=3661433

Paredes, M. (2017). Resultados de la evaluación a las habilidades lectoras de los estudiantes de tercer grado en 2016. Panamá.

Preilowski, B., \& Matute, E. (2011). Diagnóstico Neuropsicológico y Terapia de los Trastornos de LecturaEscritura (Dislexia del Desarrollo). Revista Neuropsicología, Neuropsiquiatría y Neurociencias, 11(1), 95-122. Retrieved from https://dialnet.unirioja.es/servlet/articulo?codigo=3640860\&info=resumen\&idioma=ENG

Rosario, K. J. (2016). Evaluación, diagnóstico y tratamiento psicopedagógico de la dislexia, disgrafía y discalculia en niños de 11 años. (Repositorio Digital de La UTMACH). Retrieved from http://repositorio.utmachala.edu.ec/handle/48000/8737

Simos, P. G., Fletcher, J. M., Bergman, E., Breier, J. I., Foorman, B. R., Castillo, E. M., ... Papanicolaou, A. C. (2002). Dyslexia-specific brain activation profile becomes normal following successful remedial training. Neurology, 58(8), 1203-1213.

Snowling, M. J., \& Hulme, C. (2012, May 1). Annual research review: The nature and classification of reading disorders - A commentary on proposals for DSM-5. Journal of Child Psychology and Psychiatry and Allied Disciplines, Vol. 53, pp. 593-607. https://doi.org/10.1111/j.1469-7610.2011.02495.x

Soriano-Ferrer, M., \& Piedra Martínez, E. (2017, January 1). Una revisión de las bases neurobiológicas de la dislexia en población adulta. Neurologia, Vol. 32, pp. 50-57. https://doi.org/10.1016/j.nrl.2014.08.003

Undheim, A. M. (2009). A thirteen-year follow-up study of young Norwegian adults with dyslexia in childhood: reading development and educational levels. Dyslexia, 15(4), 291-303. https://doi.org/10.1002/dys.384

Urquijo, S., Coni, A. G., \& Fernandes, D. (2015). Relationship between reading and socioeconomic level in Argentine children. Avances En Psicologia Latinoamericana, 33(2), 301-316. https://doi.org/10.12804/ap133.02.2015.09

Wechsler, D. (2007). WISC-IV: Escala de Inteligencia de Wechsler para Niños-IV (2a ed.). Madrid: TEA Ediciones. 